\title{
Chronic calcium pyrophosphate crystal inflammatory arthritis induced by extreme hypomagnesemia in short bowel syndrome
}

\author{
Markus Hahn ${ }^{1}$, Martin Raithel ${ }^{1 *}$, Alexander Hagel ${ }^{1}$, Teresa Biermann ${ }^{3}$ and Bernhard Manger ${ }^{2}$
}

\begin{abstract}
Background: Short bowel syndrome (SBS) may induce a plethora of clinical symptoms ranging from underweight to nutrient-, vitamin- and electrolyte deficiencies. The objective of this case report is to illustrate how demanding the management of a 60 year old patient with SBS and recurrent joint attacks was for different medical disciplines.

Case presentation: The patient with SBS presented with a body mass index of $16.5 \mathrm{~kg} / \mathrm{m}^{2}$ after partial jejunoileal resection of the small intestine with a six year long history of recurrent pain attacks in multiple peripheral joints, chronic diarrhoea and food intolerances. Pain attacks occurred 4-5 times a week with a median consumption of $15 \mathrm{mg}$ prednisone per day. The interdisciplinary workup after several gastroenterologic, rheumatologic, radiologic, psychiatric and orthopedic consultations is shown including successful treatment steps.

Clinical diagnosis revealed no systemic inflammatory disease, but confirmed extreme hypomagnesemia ( $0.2 \mathrm{mmol} / \mathrm{l})$ after reproducible pathological magnesium resorption tests as causative for chronic calcium pyrophosphate crystal inflammatory arthritis (pseudogout, chondrocalcinosis).

Multidisciplinary treatment included application of colchicines, parenteral nutrition and magnesium substitution, antiperistaltic agents and avoidance of intolerant foods. Normalization of magnesium levels and a marked remission of joint attacks were achieved after six months with significant reduction of prednisone to $1.5 \mathrm{mg} /$ day.
\end{abstract}

Conclusion: Despite the rarity of this condition, it is important to know that hypomagnesaemia may be associated with calcium pyrophosphate crystal inflammatory arthritis (chondrocalcinosis) and that SBS patients may be prone to develop extreme hypomagnesaemia causing recurrent joint attacks without systemic inflammation.

Keywords: SBS, Hypomagnesemia, Chondrocalcinosis, Pseudogout, CPPD

\section{Background}

Symptoms of short bowel syndrome (SBS) with mild, moderate or severe intestinal failure and/ or associated metabolic complications are estimated to occur if less than $200 \mathrm{~cm}$ of small bowel have been retained [1-3]. It has been reported to show a wide spectrum of potential complications like underweight, food intolerance, osteoporosis, steatorrhea, chologenic diarrhea with loss of bile salts, electrolytes and vitamins as well as megaloblastic anemia or renal calcium oxalate stone formation etc. [1-3]. Although SBS patients are often primarily referred to gastroenterological or nutritional hospitals, several other medical disciplines may be involved

\footnotetext{
* Correspondence: martin.raithel@uk-erlangen.de

${ }^{1}$ Department of Medicine I, Gastroenterology, University Erlangen-Nürnberg, Ulmenweg 18, Erlangen 91054, Germany

Full list of author information is available at the end of the article
}

because of extraintestinal affections or complications like hormonal dysbalances, neurological symptoms (polyneuropathy), psychiatric or psychosocial co-morbidities (anxiety, chronic fatigue and depression) or orthopedic complications (osteoporosis) etc. Thus, often only an interdisciplinary approach may be helpful to manage patients with SBS, and at least, each patient has to be treated individually, based on more or less conserved intestinal functions or manifested deficiencies [1-3]. The present case demonstrates how difficult clinical challenges can be in SBS and shows how intestinal resection may lead to severe metabolic consequences, recurrent joint attacks and food intolerances.

\section{Case presentation}

A 60 year old man presented with a 30 year history of short bowel syndrome (SBS) caused by jejunoileal 
resection of $2 / 3$ of the ileum in 1980 after abdominal trauma. The bodyweight was very low (53 kg) with a body mass index (BMI) of $16.5 \mathrm{~kg} / \mathrm{m}^{2}$. After abdominal resection he had had intravenous nutrition for some years in the 1980s, but this could be finished after successful intestinal adaptation.

At presentation in 2010, the patient complained primarily of recurrent episodes of joint pain predominantly in shoulders, knees, and ankles. He took $10-20 \mathrm{mg}$ prednisone per day and sometimes a non-steroidal analgesic to cope with the articular pain. Six years ago he had experienced an episode of severe arthritis of the knees rendering him immobile. Arthroscopy at that time revealed highly active synovitis. Back then the patient suffered from progressive dysthymia, slept long into the day, developed reduced activity and self-confidence. He complained of anxiety, tremor, restlessness and impaired concentration and was diagnosed among others by Hamilton Depression Rating Scale as having a major depressive episode [4,5]. These neuro-psychiatric symptoms had been resistant to treatment with duloxetine, amitriptyline and pregabaline for nearly two years.

Further on he reported significant underweight $\left(\mathrm{BMI}=16.5 \mathrm{~kg} / \mathrm{m}^{2}\right)$, muscular weakness, 3-4 pasty stools a day and suffered from recurrent abdominal discomfort, distension and pain. He had further significantly restricted oral nutrition because of malabsorption for some foodstuffs, lactose, fat, vitamin- and electrolyte supplements. His physical and psychological efficiency was considerably reduced.

\section{Diagnostic and therapeutic procedures}

Physical examination yielded signs of malnutrition, sarcopenia and underweight. Otherwise there were no irregularities. Electrocardiography was normal without signs of arrhythmia or elongation of intervals.

Further additional normal diagnostic tests included:

- Serum electrophoresis

- Total serum IgE and food specific IgE

- Folic acid and vitamin B6

- Anti nuclear antibodies

- Deoxyribonucleic acid antibodies

- Immune complexes

- Ferritin

- Transabdominal sonography

Systemic and gastrointestinal symptoms were assessed by the Erlangen Score for food intolerances and it showed a moderate to severe clinical disease activity with 25 points (normal $<5$, Table 1) [6]. The patient complained about pain upon active and passive motion of both knees and ankles.
While blood count and inflammatory parameters were inconspicuous, serum levels of $\mathrm{Mg}$ were extremely low (0.2 $\mathrm{mmol} / \mathrm{l} ; \mathrm{n}$ 0.7-1.1) in repeated tests, causing symptoms of muscle weakness, restlessness and paraesthesias.

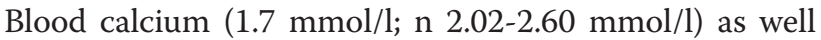
as vitamin $\mathrm{D}(12 \mathrm{ng} / \mathrm{ml}, \mathrm{n} \quad 16-70 \mathrm{ng} / \mathrm{l})$ were also depleted with clinically manifest osteoporosis and multiple fractures of vertebrae. Further supplementary laboratory data is shown in Table 2 with low serum protein levels $(58.3 \mathrm{~g} / \mathrm{l} ; \mathrm{n} 61-81 \mathrm{~g} / \mathrm{l})$ and a reduced zinc level $(64 \mu \mathrm{g} / \mathrm{dl} ; \mathrm{n} 70-120 \mu \mathrm{g} / \mathrm{dl})$.

Uric acid was normal $(5.8 \mathrm{mg} / \mathrm{dl})$. Renal loss of $\mathrm{Mg}$ was ruled out through normal values of daily urinary $\mathrm{Mg}$ excretion and fractional clearance of $\mathrm{Mg}$. To prove malabsorption as the origin of $\mathrm{Mg}$ depletion, a $\mathrm{Mg}$ resorption test was performed twice with $750 \mathrm{mg}$ magnesium oxide (18.4 mmol Mg, Magnetrans forte $150 \mathrm{mg}$, Stada GMBH, Bad Vilbel, Germany). At the time point 0 minutes the patient took 5 tablets with $250 \mathrm{ml}$ water and at the time points 60, 120 and 240 minutes the serum magnesium levels were determined. No uptake could be observed during the period of 240 minutes at a serum level of 0.2 $0.3 \mathrm{mmol} / \mathrm{l}$ (n 0.7-1.1). Hypomagnesemia as an adverse side effect of pharmacologic treatment was not found, as the patient had not taken any drugs known to affect Mghomeostasis. Hemochromatosis and hyperparathyroidism as a potential reason for CPPD deposition were ruled out by normal values of ferritin, transferrin saturation and parathyroid hormone (Table 2).

Furthermore, bacterial colonization of the remnant parts of the ileum could be excluded through a hydrogen breath test with normal fasting $\mathrm{H}_{2}$ concentrations and no premature increase of $\mathrm{H}_{2}$ gas exhalation.

Functional testing of blood leucocytes showed clearly increased leukotriene production in response to acetylsalicylic acid, characteristic of NSAID (non steroidal anti inflammatory drugs) intolerance [6,7]. This finding may explain some types of food intolerances reported from the patient which contain salicylates, but also benzoic acid, tartrazine etc. and may be causative for intolerance of some vitamin and electrolyte supplements $[6,8]$.

Endoscopic-histologic investigation by double balloon enteroscopy until $135 \mathrm{~cm}$ aborally showed no inflammatory, collagenous or lymphocytic lesions in the duodenum, jejunum or ileum. No villous atrophy was found and only discrete mild inflammatory infiltrates were focally seen within the small bowel. From enteroscopic and radiologic evaluation the length of the remaining small bowel was estimated to be around $185-195 \mathrm{~cm}$. Subtle atrophy and a slightly spotted erythema were discovered in the antrum of the stomach.

Rheumatologic examination revealed pain but no actual swelling in knees and ankles. Sonographic imaging showed linear calcification within the hyaline joint 
Table 1 Clinical parameters and score activities of the patient

\begin{tabular}{lcc}
\hline Parameter & Before treatment & $\mathbf{6}$ months after treatment \\
\hline Body weight $(\mathrm{kg})$ & 52 & 59 \\
\hline Body mass index $\left(\mathrm{kg} / \mathrm{m}^{2}\right)$ & 16.4 & 18.0 \\
\hline Stool frequency $($ stools/day) & $4-6$ & $1-2$ \\
\hline Disease activity by food intolerance score $(\mathrm{n}=$ points) & 25 & 5 \\
\hline Disease activity (joint attacks/week) & $4-5$ & none \\
\hline Consumption of prednisone $(\mathrm{mg} /$ day) & 15 & 1.5 \\
\hline Number of painful joints & 4 & 0 \\
\hline Quality of life & Severe reduction & Slight reduction \\
\hline $\begin{array}{l}\text { Clinical parameters and score activities of the patient with SBS, extreme hypomagnesemia and calcium pyrophosphate crystal inflammatory arthritis } \\
\text { (chondrocalcinosis) at the time of diagnosis and } 6 \text { months after intense interdisciplinary treatment. }\end{array}$
\end{tabular}

cartilage in both knees (see Figure 1). Radiographic results also confirmed the presence of symmetrical chondrocalcinosis in knees and ankles characteristic for CPPD deposition (see Figure 2). Aspiration of synovial fluid could not be performed in absence of effusions.

After exclusion of other inflammatory or autoimmune diseases and in view of the above mentioned clinical and radiological signs, oligoarticular chronic CPPD crystal inflammatory arthritis due to hypomagnesemia induced by SBS was diagnosed.

\section{Interdisciplinary treatment regimen significantly} improved disease course

An interdisciplinary regimen based on the following principles was established:

1. According to the EULAR recommendations [9] for the management of chronic CPPD crystal inflammatory arthritis a therapy with low-dose colchicine $(1 \mathrm{mg} / \mathrm{d})$ and prednisone $(5 \mathrm{mg} / \mathrm{d})$ was established. Due to the diagnosed non-steroidal drug

Table 2 Laboratory data for the patient

\begin{tabular}{|c|c|c|}
\hline Parameter & Before treatment & 6 months after treatment \\
\hline Hemoglobin $(12-16 \mathrm{~g} / \mathrm{dl})$ & 11.8 & 13.1 \\
\hline Leucocytes (4 - 10000/ $\mu \mathrm{l})$ & 8600 & 7100 \\
\hline Thrombocytes $(140-400000 \mu \mathrm{l})$ & 354000 & 295000 \\
\hline$\overline{\mathrm{ESR}}$ & $7 / 9$ & $4 / 6$ \\
\hline CRP mg/l $(<5)$ & 0.4 & 1.1 \\
\hline Proteins g/l (66-83) & 58.3 & 66.1 \\
\hline Albumin $\mathrm{g} / \mathrm{l}(35-55)$ & 41.1 & 39.4 \\
\hline Sodium mmol/l (135-145) & 138 & 142 \\
\hline Potassium mmol/l $(3,6-4,8)$ & 3.4 & 4.1 \\
\hline Magnesium, mmol/l (0.7-1.1) & 0.2 & 0.6 \\
\hline Calcium, mmol/l (2.1-2.7) & 1.7 & 2.2 \\
\hline Phosporus mg/dl (2.5-4.5) & 3.6 & 3.5 \\
\hline Transferrin saturation,\% (16-45\%) & 37.5 & 36.4 \\
\hline Uric acid mg/dl (3.4-7) & 5.8 & 5.8 \\
\hline Serum creatinine mg/dl (0.84-1.25) & 1.1 & 1.06 \\
\hline Urea mg/dl (17-43) & 51 & 58 \\
\hline Zinc, $\mu \mathrm{g} / \mathrm{dl}(72-175)$ & 64 & 89 \\
\hline Triglycerides mg/dl (<200) & 312 & 171 \\
\hline Cholesterol mg/dl $(<200)$ & 143 & 147 \\
\hline $250 \mathrm{H}$ vitamin D, ng/ml (30-70) & 12 & 38 \\
\hline Vitamin B12 (200 - 1100 pg/ml) & 151 & 241 \\
\hline Parathormone pg/ml (10-65) & 11.3 & 15 \\
\hline
\end{tabular}

Laboratory data for the patient with SBS, extreme hypomagnesemia and calcium pyrophosphate crystal inflammatory arthritis (chondrocalcinosis) at the time of diagnosis and 6 months after intense interdisciplinary treatment. 

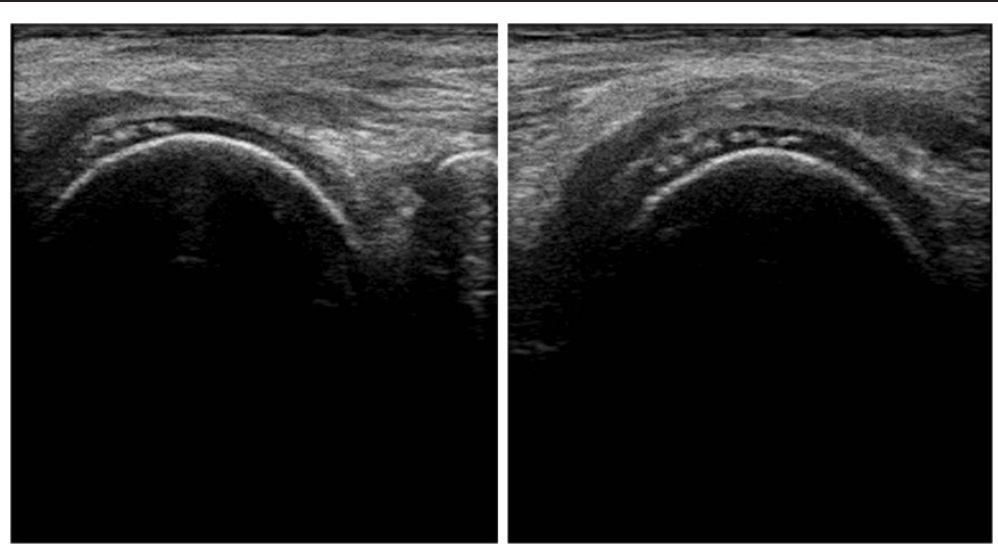

Figure 1 Sonographic detection of CPPD inflammatory arthritis (chondrocalcinosis) in the femoral condyles of both knees in SBS with extreme hypomagnesemia.

intolerance, this group of analgesics was no longer applied [6-8]. For occasional periods of analgetic treatment, tramadol or metamizol were taken.

2. Parenteral supplementary nutrition was administered monthly for six months with regular infusions of trace elements, electrolytes, Mg, vitamin B12, glutamine and lipids (For trends of laboratory data see Table 2).

3. Further nutritional advice was given to the patient to improve oral nutrition. He was especially taught to consume high caloric liquid foods, foods rich in $\mathrm{Mg}$, lipids (mostly medium-chain triglycerides), vitamin D and proteins (e.g. curds with fat content of $20-40 \%$ ).

4. Further oral trace elements, folic acid and Tinctura opii normata (containing 0.9-1.05\% morphine, 0.1\% codeine and 0.3\% thebaine (Maros $\mathrm{GmbH}$, Fürth, Germany) as an antiperistaltic agent were administered orally. Pancreatic enzymes were prescribed to increase digestive capacity of the gastrointestinal tract, as underweight, multiple food
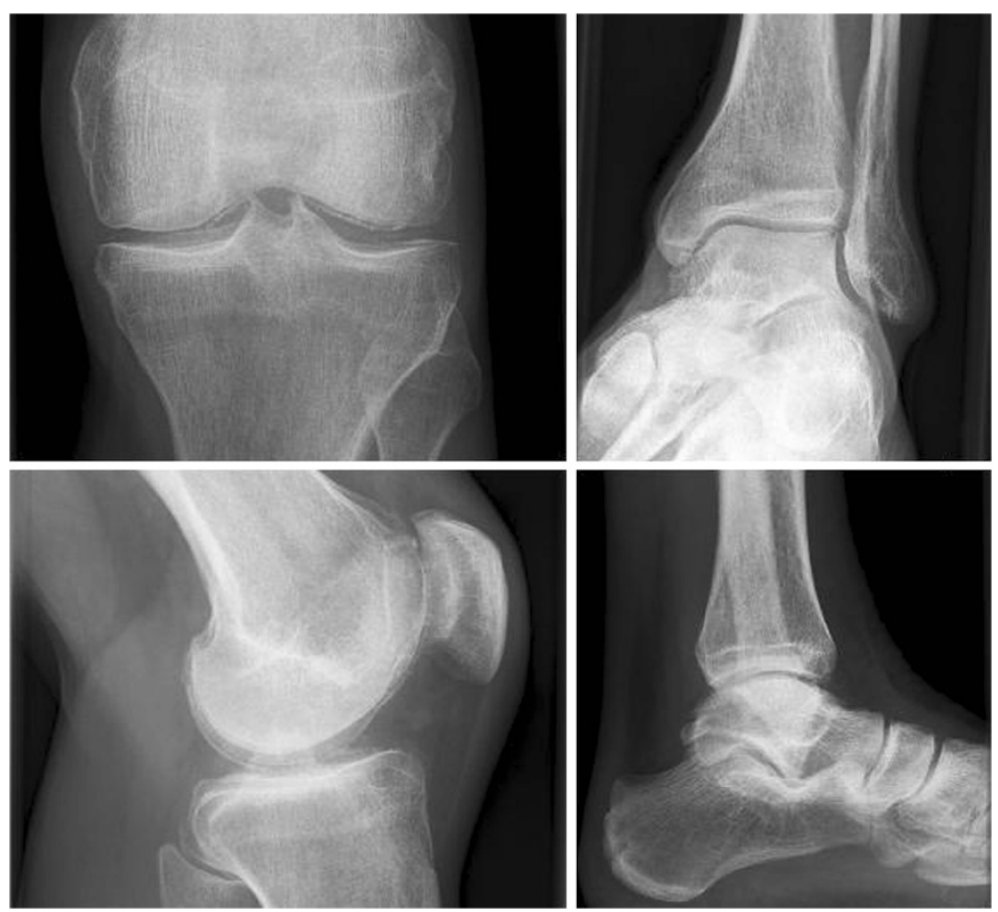

Figure 2 Radiologic findings of CPPD inflammatory arthritis (chondrocalcinosis) with tiny calcification of menisci and of hyaline cartilage in knees and ankles in SBS with extreme hypomagnesemia. 
intolerances with accelerated intestinal transit time and fat intolerance were present along with pasty stools. Accelerated transit time was suspected as one cause to reduce normal pancreatic digestion of foods, resulting in fat intolerance and abdominal discomfort.

5. According to the known intolerances, foodstuffs containing lactose or salicylates had to be excluded from the schedule [6,7] and lactase supplementation was implemented.

6. The psychiatric condition was treated with a medication regime comprising duloxetine, low dose amitriptyline and also intermittently pregabaline in various dosages as well as supportive psychotherapy.

Over the course of treatment blood levels of Mg normalized within six months (Table 2). The rate of joint attacks decreased remarkably from 4-5 times a week to approximately only one attack per month, leading to an improved quality of life which enabled the patient to get back into work. After one year of treatment the prednisone dose could be reduced to $1.5 \mathrm{mg}$ per day. Interestingly, the above mentioned neuro-psychiatric symptoms as well as sleep disturbances resolved after a few weeks of magnesium and nutrients supplementation while applying an interdisciplinary treatment regimen, but only a moderate effect on the perception of pain could be accomplished. With the gradual improvement of the patients' mind, resolution of depressive symptoms and increase of daily activities, several psychiatric consultations confirmed remission of the depressive episode. Thus, initially required drugs like duloxetine, amitryptiline etc. could be finished in the long-term follow-up and today the patient only uses benzodiazepine on demand in the case of problems getting to sleep.

\section{Discussion}

Patients may develop a plethora of clinical symptoms in SBS, when their remaining length of functional gut is insufficient for adequate absorption. Therefore, a supplementation of nutrients, water and electrolytes is usually required to maintain health and growth, respectively [10]. This case report describes a patient with extreme hypomagnesaemia and consecutive chronic CPPD crystal inflammatory arthritis due to a long history of SBS, which was inadequately supplemented and treated over years. Despite arthroscopy six years before CPPD deposition remained unrecognized. In the end, multiple current diagnostic procedures, including magnesium resorption test, revealed hypomagnesaemia as the most likely explanation for CPPD deposition. Other associated disorders such as hyperparathyreoidism, renal Mg loss and hemochromatosis were ruled out.

Since Mg-absorption testing demonstrated no resorption of $\mathrm{Mg}$ due to shortened small intestinal length with increased peristalsis because of lactose, fat and NSAID intolerance, the malabsorptive state and the SBS were considered as the reason for this extreme $\mathrm{Mg}$ deficiency. Hypomagnesaemia is a common feature of SBS. Fatty acids derived from either bacterial fermentation of malabsorbed carbohydrates or high dietary fat intake (long-chain triglycerides) bind $\mathrm{Mg}$ and prevent absorption [10]. Another contributing factor for Mg deficiency in SBS is hyperaldosteronism induced by loss of water and salt which increases renal wasting of $\mathrm{Mg}$ [11]. In addition, oral supplementation of $\mathrm{Mg}$ is known to cause intestinal motility and diarrhea, which can further compromise adequate $\mathrm{Mg}$ absorption. Finally, our patient had reduced 1,25-hydroxy-vitamin D levels, which further hampers normal jejunal Mg absorption. Thus, our patient had several pathophysiologic mechanisms contributing to hypomagnesaemia (See Figure 3 for

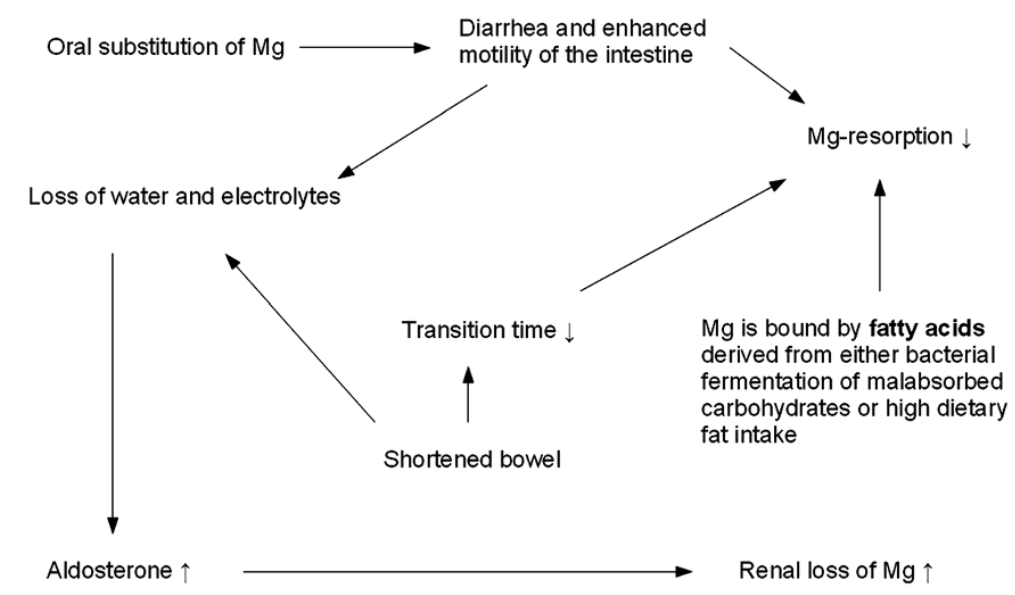

Figure 3 Pathophysiology of hypomagnesaemia in SBS. 
illustration). Therefore, parenteral substitution of $\mathrm{Mg}$ along with other nutrients was required to assure a sufficient $\mathrm{Mg}$ supply [10].

$\mathrm{Mg}$ is a divalent cation mostly located intracellularly. It plays an important role in enzymatic processes. In healthy individuals homeostasis of $\mathrm{Mg}$ is regulated effectively, as sufficient mechanisms in the gastrointestinal tract and the kidney exist. However, if these mechanisms are affected in underlying disease, $\mathrm{Mg}$ depletion can cause a wide range of symptoms such as neuromuscular manifestations (muscular cramps, vertigo, tetany), cardiac and vascular manifestations (arrhythmias, hypertension), metabolic sequels and psychiatric manifestations (depression, fatigue) $[11,12]$.

As seen in this case, severe $\mathrm{Mg}$ depletion resulted in CPPD deposition with clinical manifestations of chronic inflammatory arthritis [13-16] and was responsible for muscular weakness, fatigue and the depressive episode.

Chondrocalcinosis, is defined as the deposition of calcium pyrophosphate dihydrate in hyaline or fibrous cartilage [14]. In radiography delicate linear calcification of the cartilage can be seen. The prevalence of chondrocalcinosis increases with age (10-15\% for people between 65 and 75 years) and is hence called sporadic in patients older than 60 years, whereas in younger individuals there are several putative underlying disorders causing CPPD deposition disease, such as hemochromatosis, hyperparathyroidism, hypomagnesemia or hypophosphatemia [14]. Although often asymptomatic, CPPD crystals can cause inflammation of joints resulting in attacks of pseudogout [17]. Typical joints affected by CPPD-induced arthritis are knees and ankles as demonstrated in our patient [14]. The association between low levels of $\mathrm{Mg}$ and CPPD deposition is not fully understood. As Mg acts as a cofactor for various phosphatases, a deficiency can lead to higher amounts of pyrophosphate, a necessary precursor for the formation of CPPD crystals [18].

The linkage between SBS and Mg depletion on the one hand, as well as between Mg depletion and CPPD deposition disease on the other hand, is known. Whereas $\mathrm{Mg}$ deficiency causing CPPD deposition is due to renal failure in most cases, such as in Bartter's or Gitelmann's syndrome $[13,18,19]$, the connection of SBS causing extreme hypomagnesemia with subsequent CPPD deposition is scarce. To our knowledge, there have been only three cases described with a definite diagnosis of CPPDinduced arthritis (chondrocalcinosis) due to SBS [20].

\section{Conclusions}

Despite the rarity of this condition, it is important to know that SBS can be connected to CPPD deposition disease (chondrocalcinosis), presenting with acute or chronic arthritis and therefore SBS patients should be thoroughly investigated and, if necessary, receive parenteral treatment for $\mathrm{Mg}$ deficiency. Colchicine presents a favorable longterm medication with steroid-sparing effect, especially in the initial period of treatment [9]. Combined with several other therapeutic steps, intestinal $\mathrm{Mg}$ resorption rate was also improved by treating associated food intolerances, administering opioids to reduce bowel transit time and correcting vitamin D deficiency.

\section{Consent}

Written informed consent was obtained from the patient for publication of this Case report and any accompanying images. A copy of the written consent is available for review by the Series Editor of this journal.

\section{Abbreviations}

SBS: Short bowel syndrome; CPPD: Calcium pyrophosphate dihydrate; Mg: Magnesium; NSAID: Non steroidal anti inflammatory drugs; BMI: Body mass index.

\section{Competing interests}

The authors declare that they have no competing interests.

\section{Authors' contributions}

Each author made substantial contributions to conception and design (MR, $B M, T B$, and $M H)$, and/or acquisition of data (MR, $M H$, and $A H)$, and/or analysis and interpretation of data $(\mathrm{MH}, \mathrm{AH}, \mathrm{MR})$. All Authors participated in drafting the article or revising it critically for important intellectual content and all authors give final approval of the version to be published.

\section{Author details}

'Department of Medicine I, Gastroenterology, University Erlangen-Nürnberg, Ulmenweg 18, Erlangen 91054, Germany. 'Department of Medicine III, Rheumatology, University Erlangen-Nürnberg, Ulmenweg 18, Erlangen 91054, Germany. ${ }^{3}$ Department of Psychiatry and Psychotherapy, University Erlangen-Nürnberg, Schwabachanlage 6, Erlangen 91054, Germany.

Received: 2 March 2012 Accepted: 19 September 2012

Published: 22 September 2012

\section{References}

1. Nightingale J, Woodward JM: Guidelines for management of patients with a short bowel. Gut 2006, 55(Suppl 4):iv1-iv12.

2. Thompson JS, Weseman R, Rochling FA, Mercer DF: Current management of the short bowel syndrome. Surg Clin North Am 2011, 91:493-510.

3. Seetharam P, Rodrigues G: Short bowel syndrome: a review of management options. Saudi J Gastroenterol 2011, 17:229-235.

4. Mclntyre RS, Rosenbluth M, Ramasubbu R, Bond DJ, Taylor VH, Beaulieu S, Schaffer A: Managing medical and psychiatric comorbidity in individuals with major depressive disorder and bipolar disorder. Ann Clin Psychiatry 2012, 24:163-169.

5. Robinson M, Oakes TM, Raskin J, Liu P, Shoemaker S, Nelson JC: Acute and long-term treatment of late-life major depressive disorder: duloxetine versus placebo. Am J Geriatr Psychiatry 2012, doi:10.1097/ JGP.0b013e31825d08f1

6. Raithel M, Zopf Y, Kimpel S, Naegel A, Molderings GJ, Buchwald F, Schultis $H W$, Kressel J, Hahn EG, Konturek P: The measurement of leukotrienes in urine as diagnostic option in systemic mastocytosis. J Physiol Pharmacol 2011, 62:469-472.

7. Schafer D, Schmid M, Gode UC, Baenkler HW: Dynamics of eicosanoids in peripheral blood cells during bronchial provocation in aspirin-intolerant asthmatics. Eur Respir J 1999, 13:638-646.

8. Raithel M, Baenkler HW, Naegel A, Buchwald F, Schultis HW, Backhaus B, Kimpel S, Koch H, Mach K, Hahn EG, Konturek PC: Significance of salicylate intolerance in diseases of the lower gastrointestinal tract. $J$ Physiol Pharmacol 2005, 56(Suppl 5):89-102.

9. Zhang W, Doherty M, Pascual E, Barskova V, Guerne PA, Jansen TL, Leeb BF, Perez-Ruiz F, Pimentao J, Punzi L, et al: EULAR recommendations for 
calcium pyrophosphate deposition. Part Il: management. Ann Rheum Dis 2011, 70:571-575.

10. Nightingale JM: Management of patients with a short bowel. World J Gastroenterol 2001, 7:741-751.

11. Rude RK: Magnesium deficiency: a cause of heterogeneous disease in humans. J Bone Miner Res 1998, 13:749-758.

12. Sartori SB, Whittle N, Hetzenauer A, Singewald N: Magnesium deficiency induces anxiety and HPA axis dysregulation: modulation by therapeutic drug treatment. Neuropharmacology 2012, 62:304-312.

13. Jones AC, Chuck AJ, Arie EA, Green DJ, Doherty M: Diseases associated with calcium pyrophosphate deposition disease. Semin Arthritis Rheum 1992, 22:188-202.

14. Announ N, Guerne PA: [Diagnosis and treatment of calcium pyrophosphate crystal-induced arthropathy]. Z Rheumatol 2007, 66:573-574. 576-578.

15. Richette P, Ayoub G, Lahalle S, Vicaut E, Badran AM, Joly F, Messing B, Bardin T: Hypomagnesemia associated with chondrocalcinosis: a cross-sectional study. Arthritis Rheum 2007, 57:1496-1501.

16. Ellman MH: Images in clinical medicine. Chondrocalcinosis and hypomagnesemia. N Engl J Med 2009, 360:71.

17. Zhang W, Doherty M, Bardin T, Barskova V, Guerne PA, Jansen TL, Leeb BF, Perez-Ruiz F, Pimentao J, Punzi L, et al: European League Against Rheumatism recommendations for calcium pyrophosphate deposition. Part I: terminology and diagnosis. Ann Rheum Dis 2011, 70:563-570.

18. Calo L, Punzi L, Semplicini A: Hypomagnesemia and chondrocalcinosis in Bartter's and Gitelman's syndrome: review of the pathogenetic mechanisms. Am J Nephrol 2000, 20:347-350.

19. Cobeta-Garcia JC, Gascon A, Iglesias E, Estopinan V: Chondrocalcinosis and Gitelman's syndrome. A new association? Ann Rheum Dis 1998, 57:748-749.

20. Richette P, Ayoub G, Bardin T, Bouvet S, Orcel P, Badran AM: Hypomagnesemia and chondrocalcinosis in short bowel syndrome. J Rheumatol 2005, 32:2434-2436.

doi:10.1186/1471-230X-12-129

Cite this article as: Hahn et al:: Chronic calcium pyrophosphate crystal inflammatory arthritis induced by extreme hypomagnesemia in short bowel syndrome. BMC Gastroenterology 2012 12:129.

\section{Submit your next manuscript to BioMed Central and take full advantage of:}

- Convenient online submission

- Thorough peer review

- No space constraints or color figure charges

- Immediate publication on acceptance

- Inclusion in PubMed, CAS, Scopus and Google Scholar

- Research which is freely available for redistribution 